\title{
ChemComm
}

Cite this: Chem. Commun., 2013, 49, 7216

Received 9th May 2013,

Accepted 3rd July 2013

DOI: $10.1039 /$ c3cc43386b

\section{Enlarged tetrasubstituted alkenes with enhanced thermal and optoelectronic properties $\dagger$}

\author{
Yang Liu, ${ }^{\text {a }}$ Yun Lv, ${ }^{a} \mathrm{He} \mathrm{Xi},{ }^{a}$ Xiying Zhang, ${ }^{a}$ Shuming Chen, ${ }^{\text {b }}$ Jacky W. Y. Lam, ${ }^{c}$ \\ Ryan T. K. Kwok, ${ }^{\mathrm{C}}$ Faisal Mahtab, ${ }^{c}$ Hoi Sing Kwok, ${ }^{\mathrm{b}}$ Xutang Tao*a and \\ Ben Zhong Tang*cde
}

www.rsc.org/chemcomm

\begin{abstract}
Enlarged tetrasubstituted alkenes with high fluorescent efficiency and good thermal stability are constructed. They exhibit luminance up to $35160 \mathrm{~cd} \mathrm{~m}^{-2}$ and efficiency of $16 \mathrm{~cd} \mathrm{~A}^{-1}$ in OLEDs.
\end{abstract}

For organic chromophores, big molecules with rigid and planar sub-structures are normally used with larger conjugation extension, which benefit most of the optoelectronic processes. ${ }^{1}$ And moreover, enlarged molecules usually have better thermal stabilities, especially higher glass transition temperatures $\left(T_{\mathrm{g}}\right){ }^{2}{ }^{2}$ which is quite critical to the performance and lifetime of the optoelectronic devices. ${ }^{3}$ However, in most of the enlarged molecules, the aggregation-caused fluorescence quenching effect is more severe than that in the small ones. ${ }^{4}$ As the molecules grow larger, the intense intermolecular interactions will induce stronger non-radiative decays. To resolve this conflict, researchers have built many enlarged molecules with twisted backbones and steric hindrances. Such kind of oligomers and dendrimers have served as prominent solid emitters in many light-emitting applications. ${ }^{5}$ Whereas, people have to suffer from tedious steps during the preparation of large dendrimers. ${ }^{6}$ We can regard this a compromise between alleviation of the notorious effect of aggregation-caused quenching and complicated synthetic procedures. $^{7}$ From the view of practical usage, it is essential to develop more available materials with both nice properties and high yield of preparation. The propeller-shaped tetrasubstituted

\footnotetext{
${ }^{a}$ State Key Laboratory of Crystal Materials, Shandong University, Jinan 250100, PR China.E-mail: liuyangicm@sdu.edu.cn, txt@sdu.edu.cn

${ }^{b}$ Center for Display Research, HKUST, Kowloon, Hong Kong, China

${ }^{c}$ Department of Chemistry, State Key Laboratory of Molecular Neuroscience, Institute of Molecular Functional Materials, The Hong Kong University of Science \& Technology, Kowloon, Hong Kong, China. E-mail: tangbenz@ust.hk ${ }^{d}$ Guangdong Innovative Research Team, SCUT-HKUST Joint Research Laboratory, State Key Laboratory of Luminescent Materials and Devices, South China University of Technology, Guangzhou 510640, China ${ }^{e}$ HKUST Shenzhen Research Institute, Nanshan, Shenzhen 518057, China $\dagger$ Electronic supplementary information (ESI) available: Experimental procedures and characterization data, TEM images, TGA thermograms, calculated molecular orbitals, CV spectrum and device configurations with energy level diagrams. See DOI: $10.1039 / \mathrm{c} 3 \mathrm{cc} 43386 \mathrm{~b}$
}

alkenes would satisfy the demand. Tetraphenylethene (TPE) and its derivatives have been widely studied and used in various fields such as organic light-emitting diodes (OLEDs) ${ }^{8}$ and fluorescent sensors. ${ }^{9}$ Possessing fantastic aggregation-induced emission (AIE) characteristics, most of the TPEs have extremely high fluorescence quantum yields in the solid state. ${ }^{10}$ And more importantly, another advantage of the tetrasubstituted alkenes is that their synthetic procedures are comparatively facile. In their structures there are several positions that can be modified, making it easy to obtain highly emitting solids of enlarged molecules using this kind of structure. ${ }^{11}$

In this report, we designed two kinds of enlarged tetrasubstituted alkenes. As shown in Scheme 1, the coplanar fluorenyl unit is introduced into the core part of the alkenes to make a larger conjugation. Another two triphenylamines (TPA) or TPEs were attached peripherally to extend the molecular size further. The molecular weights of the two compounds are around one thousand and the whole molecules contain only rigid aromatic building blocks to maintain a high thermal stability. The synthetic procedures were quite facile and with high yields. Via two steps of McMurry and Suzuki coupling reactions, we can get the final products with yields beyond $85 \%$. The intermediate and the final products were characterized spectroscopically and gave satisfactory analysis data corresponding to their structures (for details of synthesis and characterization data see ESI $\dagger$ ).

Although bearing enlarged molecular size, the two dyes can dissolve readily in common organic solvents such as chloroform and THF. They are weakly or even non-emissive when

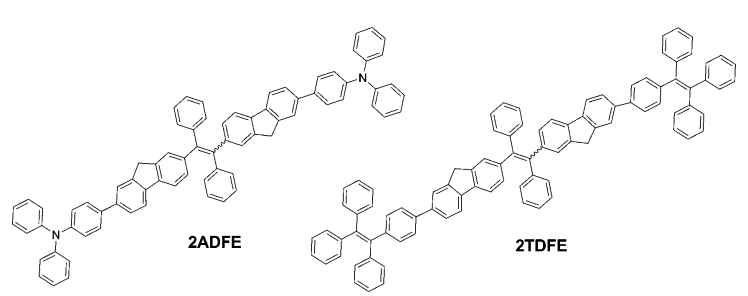

Scheme 1 Chemical structures of the two enlarged tetrasubstituted alkenes. 
molecularly dissolved in the solutions. As shown in Fig. 1, in THF solution 2ADFE has a weak emission at the short wavelength of $\sim 420 \mathrm{~nm}$, and the solution seems violet blue under UV irradiation. Upon adding water, the emission band at this wavelength decreases in intensity and disappears after the water fractions are larger than 50\%. Meanwhile, another emission at the longer wavelength of $510 \mathrm{~nm}$ appears and goes up rapidly along with the increase of the water fractions. The photoluminescence (PL) behavior of 2ADFE is quite similar to that of the polymer consisted of TPE and TPA which also retains a weak emission band at $410-420 \mathrm{~nm}$ in solution state. ${ }^{11}$ Here we regarded that owing to the extended conjugation length of 2ADFE, the intramolecular rotations around only the one alkene double bond cannot completely deactivate the excited states of the molecule via rotational energy relaxation channels. Thus a portion of the fluorescence from the TPA chromophores releases. In contrast, the molecular weight of 2TDFE is even larger, but its fluorescence in THF is completely quenched, because there are three double bonds around which the intramolecular rotations occur to consume the excited state energy. Upon adding the nonsolvent water into THF solutions of both compounds, more and more dissolved molecules start to aggregate, being accompanied by luminescence promptly. The TEM images and the electron diffraction patterns shown in Fig. S7 (ESI $\dagger$ ) proved that the aggregates are of nanodimensions and the aggregates are amorphous or with poor crystallinity. As shown in Fig. 1, in the final aggregation state with 95\% water fraction, 2ADFE shows an emission at $510 \mathrm{~nm}$ with a 50 times intensity enhancement to its original value; while 2TDFE shows a $500 \mathrm{~nm}$ emission with an enhancement of more than 100 times. From the excitation and absorption spectra (Fig. S8 and S9, ESI $\dagger$ ), we believe that the fluorescences in both solution and aggregate come from the excited molecular monomer because there is no appearance of new absorption and excitation bands. The photographs given in the insets of Fig. 1 clearly show the change from the nonemissive to emissive nature of the molecular species and aggregate particles. Being alike in the aggregate state, the thin films of 2ADFE and 2TDFE are also highly emissive. With the PL peaks of 511 and $500 \mathrm{~nm}$, their fluorescence quantum yields can both reach $100 \%$. The transient decay analyses in solution and in solid state were also implemented, the decay lifetime in solution is much shorter than that in aggregate (Fig. S10, ESI $\dagger$ ).

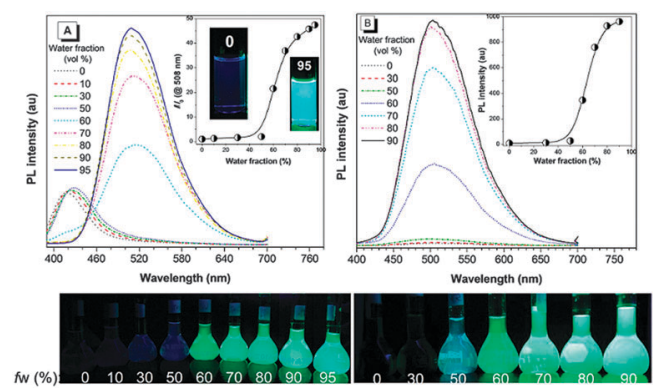

Fig. 1 PL spectra of (A) 2ADFE and (B) 2TDFE in THF-water mixtures with different water fractions $\left(f_{\mathrm{w}}\right)$. Insets in $(\mathrm{A})$ and $(\mathrm{B})$ gave the plots of PL intensity ratios $\left(I / I_{0}\right)$ and values versus the solvent compositions; the pictures are the fluorescent images of 2ADFE and 2TDFE in THF-water mixtures with different $f_{w}$.
Generally speaking, organic compounds with large molecular weight and bulky aromatic substituents normally have good thermal stabilities, especially high $T_{\mathrm{g}}$. The thermal properties of 2ADFE and 2TDFE are investigated by thermo-gravimetric (TGA) and differential scanning calorimetry (DSC) analyses. The TGA thermograms given in Fig. S11 (ESI $\dagger$ ) show decomposition temperatures of $458{ }^{\circ} \mathrm{C}$ and $484{ }^{\circ} \mathrm{C}$ for 2ADFE and 2TDFE respectively. The DSC thermograms of the two compounds are shown in Fig. S12 (ESI + ). On both the cooling and second heating cycle, we can observe a smooth step in the heat flow. For 2ADFE, its $T_{\mathrm{g}}$ locates at $163{ }^{\circ} \mathrm{C}$; for 2TDFE with TPE as terminal groups, its $T_{\mathrm{g}}$ reaches $220{ }^{\circ} \mathrm{C}$. Because materials working near or above $T_{\mathrm{g}}$ are prone to morphology changing, the heat generated during the electro-luminescence process could affect the material morphology and further degrade the device performance. Here the $T_{\mathrm{g}}$ of the two compounds are much higher than that of the commonly used optoelectronic materials, such as the well-known hole-transporting materials NPB $\left(98{ }^{\circ} \mathrm{C}\right)$ and TPD $\left(63^{\circ} \mathrm{C}\right) .^{3,12}$

As known from our previous studies, the TPA-containing 2ADFE should possess a high lying HOMO level and can act as a hole-transporting material because of the nitrogen centres. ${ }^{8 a, 11}$ Theoretical calculations performed by using a DFT/B3LYP/ 6-31G(d) method on 2ADFE reveal a severely twisted structure and a typical intramolecular charge transporting property (Fig. S13, ESI $\dagger$ ). The electrochemical properties of 2ADFE are measured by cyclic voltammetry (Fig. S14, ESI $\dagger$ ). It exhibits a similar voltammogram to that of other TPA-containing TPE oligomers, with a HOMO level derived from the onset oxidation potential to be $5.3 \mathrm{eV}$, close to that of NPB. The LUMO level obtained by subtraction of the optical band gap energy from the HOMO value is about $2.5 \mathrm{eV}$. Thus for $2 \mathrm{ADFE}$, according to the energy level diagrams shown in Fig. S15 (ESI $\dagger$ ), its HOMO and LUMO are quite matching to the energy level of the electrontransporting layer (ETL) and the work function of the anode. Two different device architectures are adopted to validate its optoelectronic behaviour. One is the typical device structure with both a hole-transporting layer (HTL) and ETL and the other one has only a ETL but no dedicated HTL.

The 2ADFE based OLEDs show strong sky blue emissions. Fig. 2 depicts the PL and EL spectra of the solid thin film. The EL spectra resemble that of the PL, and whether including a dedicated HTL or not does not affect their emission wavelength, suggesting that the recombination region remains unvarying in the 2ADFE layer. The turn-on voltage $\left(V_{\text {on }}\right)$ of both the devices is as low as $3.4 \mathrm{~V}$. After extension of the molecular conjugation, the performance is much improved compared with that of its shorter analogues. ${ }^{8 a}$ Although the maximum brightness $\left(L_{\max }\right)$ of the device with simple structure (i.e., no dedicated HTL) is inferior to that of the one with an NPB HTL, $\left(19100 \mathrm{~cd} \mathrm{~m}^{-2}\right.$ versus $25550 \mathrm{~cd} \mathrm{~m}^{-2}$ ) the brightness at the same current density is comparable. Moreover, as shown in Fig. 2B, owing to a smaller current density under the same bias, the device without HTL exhibits superior efficiencies in both current efficiency $\left(\eta_{\mathrm{C}}\right)$ and power efficiency $\left(\eta_{\mathrm{P}}\right)\left(13.7 \mathrm{~cd} \mathrm{\textrm {A } ^ { - 1 }}\right.$ versus $13.2 \mathrm{~cd} \mathrm{~A}^{-1}$ and $11.3 \mathrm{~lm} \mathrm{~W}^{-1}$ versus $11.0 \mathrm{~lm} \mathrm{~W}^{-1}$ ). This means that 2ADFE works well as both a light-emitting 

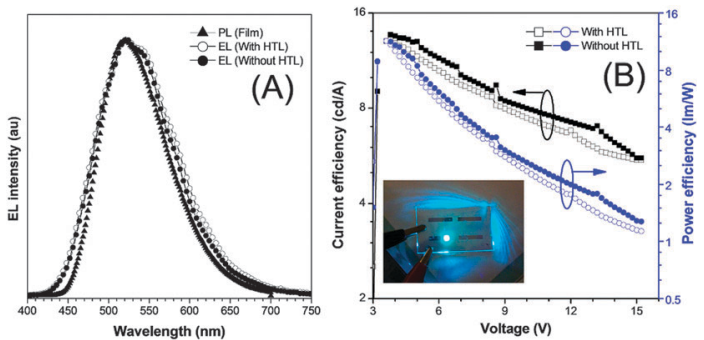

Fig. 2 (A) PL spectrum of thin film of 2ADFE and EL spectra of the devices with and without a dedicated hole-transporting layer; (B) power and current efficiencies versus applied voltage of the corresponding EL devices. The inset picture shows a working device under $8 \mathrm{~V}$ bias.
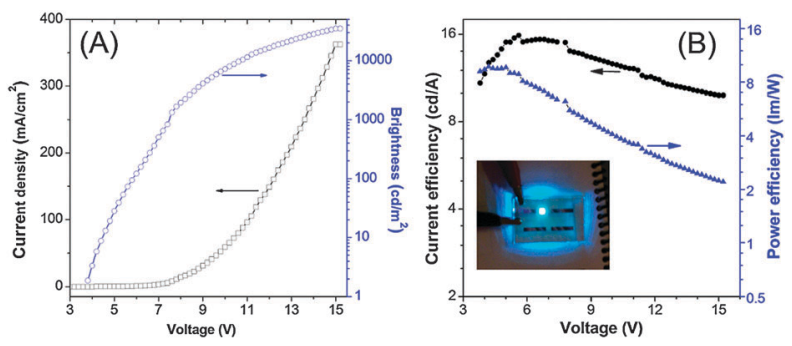

Fig. 3 Current density-voltage-luminance characteristics (A) and power and current efficiencies versus applied voltage (B) of 2TDFE device.

and hole-transporting material, which is helpful for simplifying the device fabrication procedure and lowering the production cost. Table S1 (ESI $\dagger$ ) provides details of the devices. Performances at 100 and $1000 \mathrm{~cd} \mathrm{~m}^{-2}$ are also provided as reference parameters for practical use. The larger conjugation of our AIE molecules has two sides of effect on the performance of OLEDs. The positive one is it brings excellent brightness and efficiencies. For comparison, an efficiency of $13.2 \mathrm{~cd} \mathrm{~A}^{-1}$ in oligofluorenes and a $9.1 \mathrm{~cd} \mathrm{~A}^{-1}$ by using a spiro-type fluorene as dopant has been achieved; ${ }^{13}$ for a deep-blue nondoped device, an efficiency of $2.63 \mathrm{~cd} \mathrm{~A}^{-1}$ is even a good record. ${ }^{14}$ Whereas the negative effect is that the color purity of our devices is far from comparable to these works.

The 2TDFE-based multilayer OLED (see footnote of Table S1 and Fig. S16 (ESI $\dagger$ ) for detailed device structure) also emits a strong sky blue light (Fig. 3). Thanks to the three tetrasubstituted alkene units in the molecular structure and a better thermal stability, the device shows even higher brightness (35 $160 \mathrm{~cd} \mathrm{~m}^{-2}$ ) and efficiencies. At a bias of $5.6 \mathrm{~V}$, its current efficiency is $15.9 \mathrm{~cd} \mathrm{~A}^{-1}$ and the external quantum efficiency $\left(\eta_{\text {ext max }}\right)$ reaches $4.9 \%$, closely approaching the theoretical limit for a singlet OLED.

In summary, by decorating a fluorenyl-containing alkene with large-size TPA or TPE groups, enlarged tetrasubstituted alkenes were constructed. This kind of architecture extends the conjugation of the molecules and meanwhile maintains a twisted conformation. The AIE characteristic of tetrasubstituted alkenes endows them with an extremely high efficiency of solid state fluorescence. The molecules benefit from good thermal stability, with $T_{\mathrm{g}}$ that reaches $163{ }^{\circ} \mathrm{C}$ and $220{ }^{\circ} \mathrm{C}$ respectively. They work well as emitters in OLEDs, with impressively high luminance up to $35160 \mathrm{~cd} \mathrm{~m}^{-2}$ and efficiency of $15.9 \mathrm{~cd} \mathrm{~A}^{-1}$ (external quantum efficiency 4.9\%) for 2TDFE. Additionally, the TPA-containing 2ADFE possesses a high lying HOMO level and can act as a multifunctional material for light-emitting and hole-transporting. The result demonstrates that the tetrasubstituted alkene is a good platform to build promising optoelectronic materials. The thermal, chemical and physical properties of the tetrasubstituted alkenes can be adjusted easily at our will.

This work was partially supported by the National Basic Research Program of China (973 Program, 2013CB834701), the SRFI Grants of HKUST (SRFI11SC03PG), and the Research Grants Council of Hong Kong (604711, HKUST2/CRF/10 and N_HKUST620/11). Y. Liu thanks the support from the Independent Innovation Foundation of Shandong University (2011TB020) and the Promotive Research Fund for Young Scientists of Shandong Province (BS2012CL020).

\section{Notes and references}

1 W. Hu, F. Ba, X. Gong, X. Zhan, H. Fu and T. Bjornholm, Organic Optoelectronics, Wiley VCH, 2013.

2 K.-T. Wong, Y.-Y. Chien, R.-T. Chen, C.-F. Wang, Y.-T. Lin, H.-H. Chiang, P.-Y. Hsieh, C.-C. Wu, C. H. Chou, Y. O. Su, G.-H. Lee and S.-M. Peng, J. Am. Chem. Soc., 2002, 124, 11576.

3 (a) Z. Yang, Z. Chi, B. Xu, H. Li, X. Zhang, X. Li, S. Liu, Y. Zhang and J. Xu, J. Mater. Chem., 2010, 20, 7352; (b) Z. Jiang, T. Ye, C. Yang, D. Yang, M. Zhu, C. Zhong, J. Qin and D. Ma, Chem. Mater., 2011, 23, 771.

4 J. R. Lakowicz, Principles of fluorescence spectroscopy, Springer, 3rd edn, 2006.

5 Y. Zou, T. Ye, D. Ma, J. Qin and C. Yang, J. Mater. Chem., 2012, 22, 23485.

6 Y. G. Wu, J. Y. Zhang and Z. S. Bo, Org. Lett., 2007, 9, 4435.

7 M. Zhu and C. Yang, Chem. Soc. Rev., 2013, 42, 4963.

8 (a) Y. Liu, S. Chen, J. W. Y. Lam, P. Lu, R. T. K. Kwok, F. Mahtab, H. S. Kwok and B. Z. Tang, Chem. Mater., 2011, 23, 2536; (b) Y. Liu, Y. Lv, X. Zhang, S. Chen, J. W. Y. Lam, P. Lu, R. T. K. Kwok, H. S. Kwok, X. Tao and B. Z. Tang, Chem.-Asian J., 2012, 7, 2424; (c) Y. Liu, S. Chen, J. W. Y. Lam, F. Mahtab, H. S. Kwok and B. Z. Tang, J. Mater. Chem., 2012, 22, 5184.

9 (a) Y. Hong, J. W. Y. Lam and B. Z. Tang, Chem. Soc. Rev., 2011, 40, 5361; (b) Y. Liu, Y. Tang, N. N. Barashkov, I. S. Irgibaeva, J. W. Y. Lam, R. Hu, D. Birimzhanova, Y. Yu and B. Z. Tang, J. Am. Chem. Soc., 2010, 132, 13951; (c) Y. Liu, Y. Yu, J. W. Y. Lam, Y. Hong, M. Faisal, W. Yuan and B. Z. Tang, Chem.-Eur. J., 2010, 16, 8433.

10 Y. Hong, J. W. Y. Lam and B. Z. Tang, Chem. Commun., 2009, 4332. 11 Y. Liu, X. Chen, Y. Lv, S. Chen, J. W. Y. Lam, F. Mahtab, H. S. Kwok, X. Tao and B. Z. Tang, Chem.-Eur. J., 2012, 18, 9929.

12 K. Naito and A. Miura, J. Phys. Chem., 1993, 97, 6240.

13 (a) Y.-M. Jeon, J.-Y. Lee, J.-W. Kim, C.-W. Lee and M.-S. Gong, Org. Electron., 2010, 11, 1844; (b) C.-G. Zhen, Y.-F. Dai, W.-J. Zeng, Z. Ma, Z.-K. Chen and J. Kieffer, Adv. Funct. Mater., 2011, 21, 699.

14 Y. Zhang, S.-L. Lai, Q.-X. Tong, M.-F. Lo, T.-W. Ng, M.-Y. Chan, Z.-C. Wen, J. He, K.-S. Jeff, X.-L. Tang, W.-M. Liu, C.-C. Ko, P.-F. Wang and C.-S. Lee, Chem. Mater., 2012, 24, 61. 